\title{
SEAMLESS MULTISCALE MODELING VIA DYNAMICS ON FIBER BUNDLES*
}

\author{
WEINAN $\mathrm{E}^{\dagger}$ AND JIANFENG LU
}

\begin{abstract}
We present a general mathematical framework for modeling the macroscale behavior of a multiscale system using only microscale models, by formulating the effective macroscale models as dynamic models on the underlying fiber bundles. This framework allows us to carry out seamless multiscale modeling using traditional numerical techniques. At the same time, they give rise to an interesting mathematical structure and new interesting mathematical problems. We discuss several examples from homogenization problems, continuum modeling of solids based on atomistic or electronic structure models, macroscale behavior of interacting diffusion, and continuum modeling of complex fluids based on kinetic and Brownian dynamics models.
\end{abstract}

Key words. multiscale modeling, seamless, fiber bundle

AMS subject classifications. 65J05 (35B27, 74B20, 74Q05, 76M25)

\section{Introduction}

In recent years, there has been a great deal of interests on designing multiscale modeling strategies that capture the macroscale behavior of a system using only microscopic models, bypassing the necessity for making ad hoc modeling assumptions [10]. In broad terms, such methods are divided into two categories, sequential coupling methods and concurrent coupling methods [1]. In sequential coupling methods, the required constitutive relations for the macroscopic model are precomputed. The macroscale model is then solved using traditional approaches. In concurrent coupling methods, the required model input to the macroscale simulation is computed "on-the-fly" as the simulation proceeds. This allows us to probe only the part of the constitutive relation which is used in the actual computation. If the constitutive relation depends on many parameters, this procedure can be much more efficient than precomputing the constitutive relation for the entire range of parameters. A very compelling example of concurrent coupling is the Car-Parrinello molecular dynamics [5].

In addition to the specific algorithms that have been developed for many individual applications, there have also been some efforts to develop a general framework for concurrent multiscale modeling. Most notable are the multi-grid methods [4], the heterogeneous multiscale methods (HMM) [8,9] and the equation-free methods [17]. While there are differences in the details, all three share the following features:

1. They use a concurrent coupling strategy.

2. They explicitly go back and forth between the macro and micro models. Note that the terminologies used are slightly different: Going from the macro to micro states is called interpolation, reconstruction and lifting in $[4,9$, 17] respectively. Likewise, going from the micro to macro states is called projection, compression and restriction.

\footnotetext{
${ }^{*}$ Received: May 12, 2007; accepted (in revised version): June 13, 2007. Communicated by Shi Jin.

${ }^{\dagger}$ Department of Mathematics and PACM, Princeton University, Princeton, NJ 08544, USA (weinan@Princeton.EDU).

${ }^{\ddagger}$ Program in Applied and Computational Mathematics, Princeton University, Princeton, NJ 08544, USA (jianfeng@math.Princeton.EDU).
} 
3. They all try to take advantage of the scale separation in the system. Time scale separation is used so that the microscale model is only computed for a short time, due to the fast equilibration of the microscale variables. Spatial scale separation is used so that the microscale model is only computed on a small window or a small computational cell.

These are the basic features that have made "on-the-fly" constitutive modeling an attractive approach.

From a practical viewpoint, the most difficult step is associated with the fact that these methods are quite far from being seamless: The requirement of going explicitly back and forth between the macro and micro models and/or states is often difficult in practical implementations. In this series of papers, we will develop a strategy for attacking these problems without the need to go back and forth between the macro and micro models. We will first focus on the simplest case of type B problems [9] for which a common mathematical structure will be developed with the help of fiber bundles, by formulating multiscale models as dynamics on the fiber bundles. In this paper, we will discuss the general formulation, and the mathematical problems that arise. In a follow-up paper [12], we will discuss how to make this formulation a practical tool. In subsequent papers, we will discuss increasingly more complex problems that require additional ideas.

We note that the relevance of the concept of fiber bundles was noted before in $[8,15]$. Other seamless multiscale modeling ideas are discussed in [22].

\section{The general structure of fiber bundle dynamics}

We start with a simple example. Consider the nonlinear homogenization problem:

$$
\partial_{t} u^{\varepsilon}=\nabla \cdot\left(a\left(u^{\varepsilon}, \frac{x}{\varepsilon}\right) \nabla u^{\varepsilon}\right) .
$$

Here $a(u, z)$ is a smooth and uniformly positive definite tensor function, which is periodic in $z$ with period $\Gamma$. For this problem, the homogenized equation takes the form:

$$
\partial_{t} U=\nabla \cdot(A(U) \nabla U),
$$

where ( $I$ is the identity matrix),

$$
A(U)=f a(U, z)\left(\nabla_{z} \chi(z ; U)+I\right) \mathrm{d} z,
$$

and $\chi(z ; U)$ is the solution of

$$
\nabla_{z} \cdot\left(a(U, z)\left(\nabla_{z} \chi+I\right)\right)=0 \quad \text { in } \Gamma
$$

with periodic boundary condition.

This example reveals the underlying fiber bundle structure of this type of multiscale problem. To be more precise, there are two fiber bundles (see Fig. 2.1): The first is a fiber bundle in the space of independent variables, for which the macroscale space-time domain of interest is the underlying base manifold, and the domains for the additional fast variable $z$ which parametrizes the local microstructure are the fibers. The second is a fiber bundle in the space of dependent variables, for which the macroscale quantities of interest, the range of the slow variables, are the base manifold, and the range of the quantities that characterize the microstructure, such as the probability distribution of the fast variables, are the fibers. 


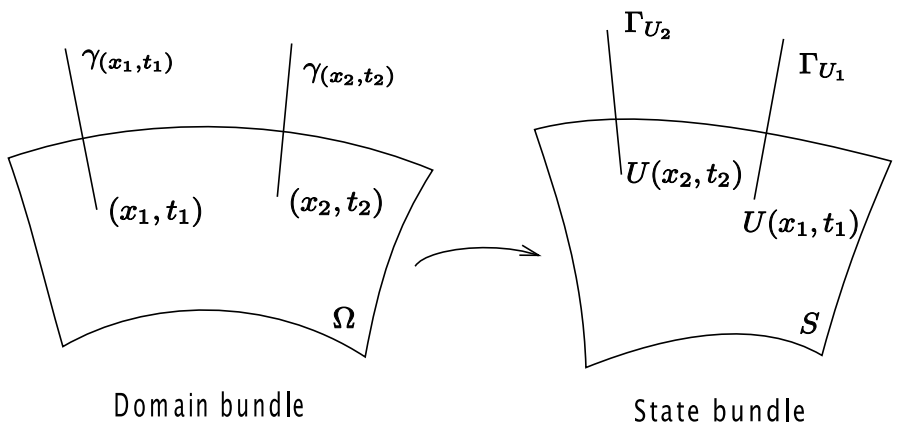

FIG. 2.1. Fiber bundle structure.

To proceed further, it is helpful to introduce some terminology. We will call the first fiber bundle the domain bundle. The base manifold in the domain bundle will be called the domain base manifold. The fibers in this bundle will be called the parametrization fibers. We will call the second fiber bundle the state bundle. The base manifold in the state bundle will be called the state base manifold. The fibers in this bundle will be called the microstate fibers.

The effective macroscopic model is a mapping from the domain bundle to the state bundle. These effective models share the following common structure. The macroscale model (2.2) maps the domain base manifold to the state base manifold, with model input given by the associated microstructure model such as (2.3). The microstructure model (2.4) maps the parametrization fibers to the microstate fibers; the values of the local macrostate (or slow variables) enter as parameters in the microstructure model.

To be more concrete, in what follows, unless otherwise noted, we will use $x$ or $(x, t)$ to denote variables on the domain base manifold, and $z$ to denote variables on the parametrization fibers. We will use $U$ to denote the variables on the state base manifold, and $u$ or $\rho$ to denote the variables on the microstate fibers. We will use $\Omega$ to denote the domain base manifold, and $\gamma_{x}$ to denote the parametrization fiber over $x, x \in \Omega$. Similarly, we will use $S$ to denote the state base manifold and $\Gamma_{U}$ to denote the microstate fiber over $U$ for $U \in S$.

Fiber bundle dynamics generally consists of the following three components:

1. A macroscale model with unspecified model input, that maps the domain base manifold to the state base manifold:

$$
L(U ; A(U))=0 .
$$

Here $U=U(x)$, and $A$ is the unspecified model input, which depends on $U$, the macrostate variables.

2. A microstructure model that maps $\gamma_{x}$ to $\Gamma_{U(x)}$, for any $x \in \Omega$ :

$$
\mathcal{L}(u ; U(x))=0 .
$$

Here $U(x)$ enters as parameters or constraints to the microscale model.

3. A formula that specifies the missing input to the macroscale model in terms of the solutions to the microstructure model:

$$
A(U)=\mathcal{A}(U, u(\cdot ; U)) .
$$


For the example discussed above, the first component is given by (2.2). The second component is given by (2.4). The third component is given by (2.3).

At this point, the fiber bundle model described above is nothing but a reformulation of homogenization or averaging theory [21]. We will see in [12] that this reformulation can be made into a very useful practical tool. However, before we proceed to the practical issues, let us discuss a few examples in which the fiber bundle structure gives rise to interesting new mathematical models.

\section{Modeling macroscopic elastic deformation of solids using atomistic models}

In traditional models of nonlinear elasticity, we formulate a variational problem of the type

$$
\min _{U} \int_{\Omega} W(\nabla U(x)) \mathrm{d} x
$$

for the elastic energy of the material, subject to certain boundary (loading) conditions. Here $x$ is the reference coordinate in the undeformed state, $U=U(x)$ is the displacement field, $y=x+U(x)$ is the position of the material point indexed by $x$ in the deformed state. The stored energy density $W$ is assumed to be a function of the deformation gradient $A=\nabla U$, and this function characterizes the elastic properties of the material. In continuum mechanics, this function is usually obtained via some constitutive assumptions with the help of symmetry considerations. The purpose of multiscale modeling is to bypass such ad hoc assumptions and obtain the function $W$ from microscopic models, which are closer to first principle models, even though they themselves are not necessarily first principle models.

It has been fully established that if thermal effects can be neglected, then the stored energy density can be obtained from atomistic models via the Cauchy-Born rule, under sharp stability conditions $[3,13,14]$. Let us assume that the atomistic potential energy takes the form:

$$
E=E\left(y_{1}, y_{2}, \cdots, y_{N}\right),
$$

where $y_{1}, y_{2}, \cdots, y_{N}$ are the positions of the atoms. A simplest, although rarely satisfactory, example of $E$ is given by

$$
E\left(y_{1}, y_{2}, \cdots, y_{N}\right)=\sum_{i \neq j} V\left(\left|y_{i}-y_{j}\right|\right),
$$

where $V$ is some empirical pair potential such as the Lennard-Jones potential. Later we will also consider the case when $E$ is given by more accurate models from quantum mechanics. Geometrically, the crystal lattice is described by its unit cell, plus some shift vectors if the lattice is a complex lattice. In the undeformed configuration, the crystal is a periodic structure. Sometimes it is more convenient to view simple lattices artificially as complex lattices. For example, the face-centered cubic lattice (FCC) is a simple lattice, but it is more convenient to view it as a complex lattice consisting of the union of the simple lattice $L$ with basis vectors $\{(a, 0,0),(0, a, 0),(0,0, a)\}$ and its shift with shift vectors $p_{1}=(a / 2, a / 2,0), p_{2}=(a / 2,0, a / 2), p_{3}=(0, a / 2, a / 2)$. The advantage of this viewpoint is that the underlying simple lattice $L$ is the standard cubic lattice. Each unit cell has four atoms, i.e., the origin and the three additional atoms at the centers of the faces connected with the origin. 
Given a matrix $A$ and the shift vectors $p=\left(p_{1}, \cdots, p_{s}\right)$, we can form a deformed unit cell by deforming the lattice vectors according to $y=x+A x$, and letting the shift vectors be $p$. This then forms a deformed lattice. Knowing the position of the atoms on the deformed lattice, we can compute the energy density according to the atomistic model, and we call this value $W(A, p)$. Strictly speaking, this value is obtained in an infinite volume limit:

$$
W(A, p)=\lim _{n \rightarrow \infty} \frac{1}{|n D|} \sum V\left(\left|y_{i}-y_{j}\right|\right),
$$

where $D$ is a smooth but otherwise arbitrary domain in space and the summation is carried out for

$$
y_{i}, y_{j} \in(n D) \bigcap \bigcup_{k=0}^{s}\left((I+A) L+p_{k}\right) .
$$

Here we have set $p_{0}=(0,0,0)$ to simplify the notation.

According to the Cauchy-Born rule, the stored energy density for the deformed crystal is given by

$$
W_{\mathrm{CB}}(A)=\min _{p} W(A, p)=W(A, p(A)),
$$

where $p=p(A)=\left(p_{1}(A), \cdots, p_{s}(A)\right)$ satisfies

$$
\nabla_{p_{j}} W(A, p(A))=0, \quad j=1, \cdots, s .
$$

Of course there is an issue whether the solution to this equation is unique. For simplicity of discussion, we will assume it is.

We can formulate this as a model on the fiber bundle. The domain base manifold is the reference configuration of the crystal. The state base manifold is $\mathbb{R}^{3 \times 3}=$ $\left\{A, A \in \mathbb{R}^{3 \times 3}\right\}$, the space of deformation gradients. The parametrization fibers $\gamma_{x}=$ $\{1,2, \cdots, s\}$ consist of the indices for the additional atoms in the unit cell. The microstructure fibers are $\Gamma_{A}=\left\{p_{1}, p_{2}, \cdots, p_{s}\right\}$. (3.1) gives the macroscale model, (3.6) gives the microstructure model and (3.5) gives the macroscale model input in terms of the microstate fiber.

This structure can be used to examine the dynamics of crystalline solids as well. The model then becomes

$$
\left\{\begin{aligned}
\partial_{t}^{2} A & =\nabla(\nabla \cdot F(A)), \\
\nabla_{p_{j}} W(A, p) & =0, \quad j=1, \cdots, s, \\
F(A) & =\nabla_{A} W(A, p) .
\end{aligned}\right.
$$

This structure also exists when the atomistic model is given by models of quantum mechanics that describe the electronic structure of the material. Let us first consider the case when the electronic structure model is given by the Thomas-Fermivon Weiszäker (TFW) model:

$$
\begin{aligned}
E\left(y_{1}, y_{2}, \cdots, y_{N}\right)= & \inf _{\rho}\left(\int \rho^{5 / 3} \mathrm{~d} y+\int|\nabla \sqrt{\rho}|^{2} \mathrm{~d} y\right. \\
& \left.+\frac{1}{2} \iint \frac{(\rho-m)(y)(\rho-m)\left(y^{\prime}\right)}{\left|y-y^{\prime}\right|} \mathrm{d} y \mathrm{~d} y^{\prime}\right) .
\end{aligned}
$$


Here $\rho$ is the electron density and $m$ is the charge distribution due to the ions,

$$
m(y)=\sum_{i=1}^{N} m^{a}\left(y-y_{i}\right),
$$

where $m^{a}$ is the pseudopotential of a single ion. $\rho$ satisfies the normalization constraint:

$$
\int \rho \mathrm{d} y=n N
$$

where we assume that each atom has $n$ valence electrons.

In this case, the structure of the fibers needs to be modified. For simplicity, we only discuss the case of a simple lattice. The parametrization fiber is $\gamma_{x}=\Gamma$, where $\Gamma$ is the undeformed unit cell, the microstate fibers are $\Gamma_{A}=\rho(\cdot)$.

Given the matrix $A$ and the electron density $\rho$, the energy density of the deformed lattice $W(A, \rho)$ can be computed by the periodic TFW model $[6,11]$ :

$$
\begin{array}{r}
W(A, \rho)=\frac{\operatorname{det}(I+A)}{|\Gamma|}\left(\int_{\Gamma} \rho^{5 / 3}(z ; A) \mathrm{d} z+\int_{\Gamma}\left|(I+A)^{-\mathrm{T}} \nabla_{z} \sqrt{\rho(z ; A)}\right|^{2} \mathrm{~d} z\right. \\
\left.\quad+\frac{1}{2} \iint_{\Gamma \times \Gamma}(\rho-m)(z ; A) G\left(z-z^{\prime} ; A\right)(\rho-m)\left(z^{\prime} ; A\right) \mathrm{d} z \mathrm{~d} z^{\prime}\right) .
\end{array}
$$

Here $m$ is the charge distribution generated by the deformed lattice

$$
m(z ; A)=\sum_{z_{i} \in L} m^{a}\left((I+A)\left(z-z_{i}\right)\right) .
$$

$G$ is the periodic Coulombic kernel [19] which is the solution of

$$
-\left((I+A)^{-\mathrm{T}} \nabla_{z}\right)^{2} G(z ; A)=4 \pi(\delta(z)-1 /|\Gamma|) \quad \text { in } \Gamma
$$

with periodic boundary condition and $\lim _{z \rightarrow 0}(G(z ; A)-1 /|(I+A) z|)=0$.

Again, the Cauchy-Born stored energy density is given by

$$
W_{\mathrm{CB}}(A)=\min _{\rho} W(A, \rho)=W(A, \rho(\cdot ; A)),
$$

where $\rho=\rho(\cdot ; A)$ satisfies

$$
\left\{\begin{aligned}
\frac{\delta}{\delta \rho} W(A, \rho(\cdot ; A)) & =\lambda \\
\int_{\Gamma} \rho(z ; A) \mathrm{d} z & =n / \operatorname{det}(I+A) .
\end{aligned}\right.
$$

Here $\lambda$ is the Lagrange multiplier for the normalization constraint. The macroscale model is still given by (3.1), while (3.15) gives the microstructure model and (3.14) gives the macroscale model input from the microstructure. In this case, the dynamic model is given by

$$
\left\{\begin{aligned}
\partial_{t}^{2} A & =\nabla(\nabla \cdot F(A)), \\
\frac{\delta}{\delta \rho} W(A, \rho(\cdot ; A)) & =\lambda \\
\int_{\Gamma} \rho(z ; A) \mathrm{d} z & =n / \operatorname{det}(I+A), \\
F(A) & =\nabla_{A} W(A, \rho) .
\end{aligned}\right.
$$


Next, we consider the case when the electronic structure model is given by the Kohn-Sham Density Function Theory (KSDFT):

$$
\begin{aligned}
E\left(y_{1}, y_{2}, \cdots, y_{N}\right)=\inf _{\left\{\psi_{\alpha}\right\}}\left(\sum_{\alpha} \int\left|\nabla \psi_{\alpha}(y)\right|^{2} \mathrm{~d} y+\int \epsilon_{x c}(\rho(y)) \mathrm{d} y\right. \\
\left.+\frac{1}{2} \iint \frac{(\rho-m)(y)(\rho-m)\left(y^{\prime}\right)}{\left|y-y^{\prime}\right|} \mathrm{d} y \mathrm{~d} y^{\prime}\right) .
\end{aligned}
$$

Here $\psi=\left\{\psi_{\alpha}\right\}_{\alpha=1}^{n N}$ is a collection of orthonormal wave functions, $\rho$ is the corresponding electron density given by $\rho(y)=\sum_{\alpha}\left|\psi_{\alpha}(y)\right|^{2}$, and $\epsilon_{x c}$ is the exchange-correlation potential with local density approximation used.

In the KSDFT case, the parametrization fiber is $\gamma_{x}=\mathbb{R}^{3 n / 2}$, where we assume that $n$ is even for simplicity; the factor $1 / 2$ is due to spin degeneracy. The microstate fibers are $\Gamma_{A}=\left\{\psi_{1}(\cdot), \psi_{2}(\cdot), \cdots, \psi_{n / 2}(\cdot)\right\}$, where $\left\{\psi_{\alpha}\right\}_{\alpha=1}^{n / 2}$ are the wave functions. The energy density $W(A,\{\psi\})$ of the deformed lattice is given by a periodic KSDFT model [11]:

$$
\begin{array}{r}
W(A,\{\psi\})=\frac{\operatorname{det}(I+A)}{|\Gamma|}\left(\sum_{\alpha=1}^{n / 2} \int_{\mathbb{R}^{3}}\left|(I+A)^{-\mathrm{T}} \nabla_{z} \psi_{\alpha}(z ; A)\right|^{2} \mathrm{~d} z+\int_{\Gamma} \epsilon_{x c}(\rho(z ; A)) \mathrm{d} z\right. \\
\left.\left.+\frac{1}{2} \iint_{\Gamma \times \Gamma}(\rho-m)(z ; A)\right) G\left(z-z^{\prime} ; A\right)(\rho-m)\left(z^{\prime} ; A\right) \mathrm{d} z \mathrm{~d} z^{\prime}\right) .
\end{array}
$$

Here, $m$ and $G$ are defined as in (3.12) and (3.13), and $\rho$ is the periodic electron density given by

$$
\rho(z ; A)=2 \sum_{z_{i} \in L} \sum_{\alpha=1}^{n}\left|\psi_{\alpha}\left(z-z_{i} ; A\right)\right|^{2} .
$$

Again, the Cauchy-Born stored energy density is given by

$$
W_{\mathrm{CB}}(A)=\min _{\{\psi\}} W(A,\{\psi\})=W(A,\{\psi(\cdot ; A)\}),
$$

where $\{\psi\}=\{\psi(\cdot ; A)\}$ satisfy

$$
\left\{\begin{aligned}
\frac{\delta}{\delta \psi_{\alpha}} W(A,\{\psi(\cdot ; A)\}) & =\sum_{\alpha^{\prime}, z_{j} \in L} \lambda_{\alpha \alpha^{\prime}, z_{j}} \psi_{\alpha^{\prime}}\left(z-z_{j} ; A\right), \\
\int \psi_{\alpha}^{*}\left(z-z_{i} ; A\right) \psi_{\alpha^{\prime}}\left(z-z_{j} ; A\right) \mathrm{d} z & =\delta_{\alpha \alpha^{\prime}} \delta_{i j} / \operatorname{det}(I+A) .
\end{aligned}\right.
$$

Here $\lambda=\left\{\lambda_{\alpha \alpha^{\prime}, z_{j}}\right\}$ are the Lagrange multipliers for the orthonormality constraints. The macroscale model is still given by (3.1), while (3.21) gives the microstructure model and (3.20) gives the macroscale model input from the microstructure. In this case, the dynamic model is given by

$$
\left\{\begin{aligned}
\partial_{t}^{2} A & =\nabla(\nabla \cdot F(A)), \\
\frac{\delta}{\delta \psi_{\alpha}} W(A,\{\psi(\cdot ; A)\}) & =\sum_{\alpha^{\prime}, z_{j} \in L} \lambda_{\alpha \alpha^{\prime}, z_{j}} \psi_{\alpha^{\prime}}\left(z-z_{j} ; A\right), \\
\int \psi_{\alpha}^{*}\left(z-z_{i} ; A\right) \psi_{\alpha^{\prime}}\left(z-z_{j} ; A\right) \mathrm{d} z & =\delta_{\alpha \alpha^{\prime}} \delta_{i j} / \operatorname{det}(I+A), \\
F(A) & =\nabla_{A} W(A,\{\psi\}) .
\end{aligned}\right.
$$




\section{Relaxational dynamics on the fibers}

The form of the macroscale models discussed so far corresponds to the BornOppenheimer adiabatic approximation in quantum mechanics, in which the electronic structure is assumed to be in the ground state given by the positions of the nuclei. In the spirit of Car-Parrinello molecular dynamics [5], we can introduce a relaxational dynamics to the electronic structure equation. More generally, we can introduce relaxational dynamics for the fibers. For example, in the TFW case, the dynamics for the microstructure becomes

$$
m \partial_{t}^{2} \rho+\eta \partial_{t} \rho=-\frac{\delta}{\delta \rho} W(A, \rho)(z)+\lambda .
$$

Here, $m$ is an artificial electron mass and $\eta$ is a relaxation parameter. $\lambda$ is the Lagrange multiplier for the normalization constraint $\int_{\Gamma} \rho(z ; A) \mathrm{d} z=n / \operatorname{det}(I+A)$. To determine the model input $F(A)$, we have

$$
0=\nabla_{A} W(A, \rho)=\partial_{A} W(A, \rho)+\frac{\delta}{\delta \rho} W(A, \rho) \partial_{A} \rho .
$$

The derivative can be evaluated using the Hellmann-Feynman theorem which needs to be generalized to this setting. For simplicity, let us denote by $g(A, \rho)$ the constraint

$$
g(A, \rho)=\int_{\Gamma} \rho(z ; A) \mathrm{d} z-n / \operatorname{det}(I+A) .
$$

The minimizer $\rho$ satisfies

$$
\frac{\delta}{\delta \rho} W(A, \rho)-\lambda \frac{\delta}{\delta \rho} g(A, \rho)=0 .
$$

Also, since $g(A, \rho)=0$ is always satisfied by the minimizer, we have

$$
\nabla_{A} g(A, \rho)=\partial_{A} g(A, \rho)+\frac{\delta}{\delta \rho} g(A, \rho) \partial_{A} \rho .
$$

Substituting (4.4) and (4.5) into (4.2), we get

$$
\nabla_{A} W(A, \rho)=\partial_{A} W(A, \rho)-\lambda \partial_{A} g(A, \rho) .
$$

In the case of the TFW model,

$$
\partial_{A} g(A, \rho)=n(I+A)^{-1} / \operatorname{det}(I+A) .
$$

Therefore, (3.16) becomes

$$
\left\{\begin{aligned}
\partial_{t}^{2} A & =\nabla(\nabla \cdot F(A)), \\
m \partial_{t}^{2} \rho+\eta \partial_{t} \rho & =-\frac{\delta}{\delta \rho} W(A, \rho)(z)+\lambda, \\
\int_{\Gamma} \rho(z ; A) \mathrm{d} z & =n / \operatorname{det}(I+A), \\
F(A) & =\partial_{A} W(A, \rho)-\lambda n(I+A)^{-1} / \operatorname{det}(I+A) .
\end{aligned}\right.
$$

Next we consider some simple examples in order to see explicitly the mathematical structure of these models. First, let us consider a one-dimensional chain with two 
alternating species of atoms $A$ and $B$ with nearest neighbor interaction. We denote by $V$ the interaction between the $A$ and $B$ atoms. We assume that the lattice constant is 1 and the shift vector now is the distance between an $A$ atom and the following $B$ atom. Then we have:

$$
W(A, p)=V(p)+V(1+A-p)
$$

Hence,

$$
\begin{aligned}
& \nabla_{A} W(A, p)=V^{\prime}(1+A-p), \\
& \nabla_{p} W(A, p)=V^{\prime}(p)-V^{\prime}(1+A-p) .
\end{aligned}
$$

We can write the dynamics as

$$
\left\{\begin{aligned}
\partial_{t}^{2} A & =\partial_{x}^{2} F(A), \\
m \partial_{t}^{2} p+\eta \partial_{t} p & =-V^{\prime}(p)+V^{\prime}(1+A-p), \\
F(A) & =V^{\prime}(1+A-p) .
\end{aligned}\right.
$$

Next we change the atomistic model to a quantum mechanical model. We consider a one-dimensional chain of a single kind of atom $A$, where each atom has one valence electron. We consider a model whose periodic energy functional is given by

$$
W(A, \psi)=\int_{0}^{1+A}\left(\left|\partial_{z} \psi\right|^{2}+V\left(\psi^{2}\right)\right) \mathrm{d} z
$$

Here $\psi$ satisfies the normalization constraint

$$
\int_{0}^{1+A} \psi^{2}(z) \mathrm{d} z=1
$$

$V$ is a general nonlinear potential. We calculate the explicit formula following (4.8). Notice that we have written the microstructure model using the coordinates in the deformed state, instead of the reference state as in (3.15) and (3.21). This choice of coordinates is more convenient in this case.

The dynamics microstructure model is given by

$$
m \partial_{t}^{2} \psi+\eta \partial_{t} \psi=\partial_{z}^{2} \psi-V^{\prime}\left(\psi^{2}\right) \psi+\lambda \psi
$$

Here $m$ is an artificial electron mass, $\eta$ is a relaxation parameter, and $\lambda$ is the Lagrange multiplier. We calculate $F(A)$ according to (4.6):

$$
F(A)=\nabla_{A} W(A, \psi)=\left|\partial_{z} \psi(0 ; A)\right|^{2}+V\left(\psi^{2}(0 ; A)\right)-\lambda \psi^{2}(0 ; A) .
$$

Notice that in the case of the quasi-static microstructure model, when $\psi(\cdot ; A)$ is the minimizer of $\min W(A, \psi)$ satisfying the constraint, i.e., if

$$
-\partial_{z}^{2} \psi+V^{\prime}\left(\psi^{2}\right) \psi=\lambda \psi
$$

then

$$
S(z)=\left|\partial_{z} \psi(z ; a)\right|^{2}+V\left(\psi^{2}(z ; A)\right)-\lambda \psi^{2}(z ; A)
$$


does not depend on $z$. However, if the model is replaced by (4.15), then this quantity is no longer independent of $z$. Therefore, in this case, it is natural to use instead

$$
\begin{aligned}
F(A) & =f\left(\left|\partial_{z} \psi(z ; A)\right|^{2}+V\left(\psi^{2}(z ; A)\right)-\lambda \psi^{2}(z ; A)\right) \mathrm{d} z \\
& =f\left(\left|\partial_{z} \psi(z ; A)\right|^{2}+V\left(\psi^{2}(z ; A)\right)\right) \mathrm{d} z-\lambda /(1+A) .
\end{aligned}
$$

Therefore, the dynamics for the whole system is

$$
\left\{\begin{aligned}
\partial_{t}^{2} A & =\nabla(\nabla \cdot F(A)) \\
m \partial_{t}^{2} \psi+\eta \partial_{t} \psi & =\partial_{z}^{2} \psi-V^{\prime}\left(\psi^{2}\right) \psi+\lambda \psi \\
\int_{0}^{1+A}|\psi(z ; A)|^{2} \mathrm{~d} z & =1 \\
F(A) & =f\left(\left|\partial_{z} \psi(z ; A)\right|^{2}+V\left(\psi^{2}(z ; A)\right)\right) \mathrm{d} z-\lambda /(1+A) .
\end{aligned}\right.
$$

5. Macroscopic behavior of interacting diffusion

Let us consider a Ginzburg-Landau lattice model for interacting diffusion. Let $L_{N} \subset L$ be a sublattice of length $N$ with periodic boundary condition and let $H_{N}$ be a Ginzburg-Landau type of Hamiltonian given by

$$
H_{N}=\frac{1}{2} \sum_{i, j \in L_{N},|i-j|=1}\left|\phi_{i}-\phi_{j}\right|^{2}+\sum_{i \in L_{N}} V\left(\phi_{i}\right),
$$

where $V$ is the potential $\left(V(\phi)=\alpha \phi^{2}+\lambda \phi^{4}\right.$ with $\lambda>0$, for example). The lattice function $\phi$ is the state variable of the lattice model. One can describe the dynamics by the stochastic differential equations

$$
\mathrm{d} \phi_{i}=\sum_{j \in L_{n},|i-j|=1} \frac{N^{2}}{2}\left(\frac{\partial H_{N}}{\partial \phi_{j}}-\frac{\partial H_{N}}{\partial \phi_{i}}\right) \mathrm{d} t-\sqrt{\frac{N^{2}}{\beta}} \mathrm{d} W_{t}(i, j) .
$$

Here $W_{t}(i, j)=-W_{t}(j, i)$ is a Wiener process associated with the bond $(i, j)$, independent for different bonds. $\beta=1 / k_{\mathrm{B}} T$ is a parameter related to temperature.

It has been proved $[16,23]$ under various assumptions that the the GinzburgLandau dynamics has a macroscopic density $U(x, t)$ satisfying the diffusion equation

$$
\partial_{t} U=\nabla \cdot(D(U) \nabla U)
$$

Here $D(U)$ is the diffusion coefficient given by the second derivative of the free energy of the microscopic models in the thermodynamic limit.

Recall the definition of free energy for the lattice model $L_{N}$ :

$$
F_{N}(U)=\frac{1}{\left|L_{N}\right|} \log \int \exp \left(-\beta H_{N}\right) \delta(\bar{\phi}-U) \mathrm{d} \phi
$$

$F_{N}$ is the ensemble average taken on the constraint manifold $\bar{\phi}=U$, where $\bar{\phi}$ is the average value of $\phi$ :

$$
\bar{\phi}=\frac{1}{\left|L_{N}\right|} \sum_{i \in L_{N}} \phi_{i}
$$


Taking the thermodynamic limit $N \rightarrow \infty, F_{N}$ has unique limit $F$, and

$$
D(U)=\frac{1}{2} \partial_{U}^{2} F(U)
$$

We can formulate this as a model on the fiber bundle. The domain base manifold is the physical space-time where the diffusion takes place. The state base manifold is $\mathbb{R}$, the space of density. The parametrization fibers $\gamma_{x, t}=L_{N}$ consist of the lattice grids. The microstructure fibers are $\Gamma_{U}=\left\{\phi_{i}\right\}_{i \in L_{N}}$. (5.3) gives the macroscale model, (5.1) gives the microscale model and (5.5) gives the input from the microstate fibers.

The integration (5.4) is difficult to evaluate directly. In practice, it is evaluated through dynamics such as the Langevin equation, molecular dynamics, or overdamped dynamics, etc. For example, if overdamped Langevin dynamics is used, the model then becomes

$$
\left\{\begin{aligned}
\partial_{t} U & =\Delta(A(U)), \\
\mathrm{d} \phi_{i} & =\sum_{j \in L_{n},|i-j|=1}\left\{\frac{1}{2 \eta}\left(\frac{\partial H_{N}}{\partial \phi_{j}}-\frac{\partial H_{N}}{\partial \phi_{i}}\right) \mathrm{d} t-\sqrt{\frac{1}{\eta \beta}} \mathrm{d} W_{t}(i, j)\right\}+\lambda \mathrm{d} t \\
A(U) & =\frac{1}{2} \frac{1}{\left|L_{N}\right|} \sum_{i \in L_{N}}\left(-\beta \frac{\partial H_{N}}{\partial \phi_{i}}\right) .
\end{aligned}\right.
$$

Here $\eta$ is a relaxational parameter, and $\lambda$ is the Lagrange multiplier given by $\lambda=\partial_{t} U$.

The effective macroscale dynamics of $(5.6)$ is

$$
\left\{\begin{aligned}
\partial_{t} U & =\Delta(A(U)) \\
A(U) & =\frac{1}{2} \partial_{U}\left(\frac{1}{\left|L_{N}\right|} \log \int \exp \left(-\beta H_{N}\right) \delta(\bar{\phi}-U) \mathrm{d} U\right) .
\end{aligned}\right.
$$

We remark that the choice of microscopic dynamics and the way to impose constraints are not unique.

\section{Kinetic and Brownian dynamics models of complex fluids}

The models discussed above all share the feature that the microstructure models on different fibers are independent of each other: the states on different microstate fibers are connected to each other only through the base manifold. This feature is quite attractive for numerical purposes since it facilitates the implementation of parallel algorithms and the localization of the microstructure models. For this reason, HMM has made a point of retaining this feature in numerical algorithms [8]. However, there are multiscale models that do not share this feature. We now discuss some examples of such models.

Let us consider kinetic models for complex fluids such as polymer fluids or liquid crystal flows [18]. In these kinetic models, besides the velocity field, we also consider a configuration distribution function $\psi$ for the conformation of the polymers or liquid crystals. The configuration distribution function is defined on the configuration space $G$. For rod-like polymers whose configuration is described by the orientation of the rods, $G$ is the space of orientations, i.e., the unit sphere $\mathbb{S}^{2}$ with opposite points identified. For bead-and-spring polymers, $G$ is the space that describes the position of the beads. In general, the kinetic models for bead-and-spring polymers are of the 
following form:

$$
\left\{\begin{aligned}
\partial_{t} U+\left(U \cdot \nabla_{x}\right) U+\nabla_{x} P & =\frac{\nu}{R e} \Delta_{x} U+\frac{1-\nu}{R e D e} \nabla_{x} \cdot \tau_{p}, \\
\nabla_{x} \cdot U & =0 \\
\partial_{t} \psi+\nabla_{x} \cdot(U \psi) & =-\nabla_{z} \cdot\left(\left(\nabla_{x}^{\mathrm{T}} U \cdot z-\frac{1}{2 D e} f(z)\right) \psi\right)+\frac{1}{2 D e} \Delta_{z} \psi, \\
\tau_{p}(x, t) & =\int_{G}(f(z) \otimes z) \psi(x, z, t) \mathrm{d} z .
\end{aligned}\right.
$$

Here $z$ are the microstate variables describing the position of beads ( $n$ vectors if we have $n$ springs for each polymer), $f(z)$ is the configurational force. If we use more than one spring to represent each polymer, $f(z) \otimes z$ means $\sum_{j} f^{(j)}(z) \otimes z^{(j)}$, where $z^{(j)}$ is the vector corresponds to the $j$-th spring. $U$ and $P$ are macroscopic velocity and pressure respectively. Dimensionless parameters $R e, D e, \nu$ are Reynolds number, Deborah number, viscosity ratio respectively. For rod-like polymers, we have in general the following kinetic models:

$$
\left\{\begin{aligned}
\partial_{t} U+\left(U \cdot \nabla_{x}\right) U+\nabla_{x} P & =\frac{\nu}{R e} \Delta_{x} U+\frac{1-\nu}{R e D e} \nabla_{x} \cdot \tau_{p}, \\
\nabla_{x} \cdot U & =0 \\
\partial_{t} \psi+\nabla_{x} \cdot(U \psi) & =\frac{1}{D e} \mathcal{R} \cdot(\mathcal{R} \psi+\psi m \times f)-\mathcal{R} \cdot\left(m \times \nabla_{x}^{\mathrm{T}} U \cdot m \psi\right), \\
\tau_{p}(x, t) & =\int_{G}((3 m \otimes m-I)-(m \times m \times f) \otimes m \\
& \left.+\frac{D e}{2}\left(\nabla_{x}^{\mathrm{T}} U: m \otimes m \otimes m \otimes m\right)\right) \psi(x, m, t) \mathrm{d} m .
\end{aligned}\right.
$$

Here $m$ is the rotation angel of the polymer and $f(m)$ is the configurational force given by external field or a mean-field interaction potential. $\mathcal{R}$ is the rotational operator given by $\mathcal{R}=m \times \nabla_{m}$. In these models, the expression for $\tau_{p}$, the polymer stress, is usually called Kramers expression [2, 7].

In the language of fiber bundles, the domain base manifold is the physical spacetime domain $\Omega$, the parametrization fibers are $\gamma_{x, t}=G$, the state base manifold is the space of velocity and velocity gradients $R^{3} \times R^{3 \times 3}$, and the microstate fibers are $\Gamma_{U, A}=\{\psi(\cdot)\}$. However, this model is different from the models we discussed before, since the microstate on different fibers are linked to each other by the convection term $\left(U \cdot \nabla_{x}\right) \psi$.

In the above form, the microstructure dynamics involved in (6.1) and (6.2) are expensive to solve, since they are Fokker-Planck equations. In practice, it is more effective to replace solving the Fokker-Planck equations by sampling:

$$
\left\{\begin{aligned}
\partial_{t} U+\left(U \cdot \nabla_{x}\right) U+\nabla_{x} P & =\frac{\nu}{R e} \Delta_{x} U+\frac{1-\nu}{R e D e} \nabla_{x} \cdot \tau_{p}, \\
\nabla_{x} \cdot U & =0 \\
\partial_{t} z_{j}+\left(U \cdot \nabla_{x}\right) z_{j} & =\nabla_{x}^{\mathrm{T}} U \cdot z_{j}-\frac{1}{2 D e} f\left(z_{j}\right)+\sqrt{\frac{1}{D e}} \dot{W}_{j}, \\
\tau_{p}(x, t) & =\frac{1}{N} \sum_{j=1}^{N} f\left(z_{j}\right) \otimes z_{j},
\end{aligned}\right.
$$


and

$$
\left\{\begin{aligned}
& \partial_{t} U+\left(U \cdot \nabla_{x}\right) U+\nabla_{x} P=\frac{\nu}{R e} \Delta_{x} U+\frac{1-\nu}{R e D e} \nabla_{x} \cdot \tau_{p}, \\
& \nabla_{x} \cdot U=0 \\
& \partial_{t} m_{j}+\left(U \cdot \nabla_{x}\right) m_{j}=\left(I-m_{j} \otimes m_{j}\right) \\
& \circ\left(-\frac{1}{D e} m_{j} \times f\left(m_{j}\right)+m_{j} \times \nabla_{x}^{\mathrm{T}} U \cdot m_{j}+\sqrt{\frac{2}{D e}} \dot{W}_{j}\right), \\
& \tau_{p}(x, t)= \frac{1}{N} \sum_{j=1}^{N}\left(3 m_{j} \otimes m_{j}-I\right)-\left(m_{j} \times m_{j} \times f\left(m_{j}\right)\right) \otimes m_{j} \\
&+\frac{D e}{2}\left(\nabla_{x}^{\mathrm{T}} U: m_{j} \otimes m_{j} \otimes m_{j} \otimes m_{j}\right),
\end{aligned}\right.
$$

where $\circ$ means the Stratonovich integral is used since the Brownian motion is on the unit sphere $\mathbb{S}^{2}$.

\section{Some practical issues}

The main advantage of the fiber bundle dynamics models is that they are now amendable to standard numerical techniques; the difficulties associated with the multiscale issues are largely removed. Compared with other multiscale strategies such as HMM, the models have the advantage that they are seamless, and therefore much easier to implement. Preliminary numerical results also indicate that the fiber bundle models are also more efficient [20]. As we discussed above, most of these fiber bundle dynamics models have the feature that the microstructure models are only linked to each other through the base manifolds. In this case, parallelization is embarrassingly simple. In other cases, developing parallel algorithms does not appear to be a difficult task either. In this regard, fiber bundle dynamics share the same feature as HMM.

From some of the examples discussed above, such as the first example, it may appear that the fiber bundle dynamics is simply a reformulation of the homogenized equation. This does not have to be the case: one can formulate fiber bundle dynamics models on the microscale problems with much less analytical understanding. The most essential ingredients are the links between the macro and micro models; the formula that expresses the macroscale data in terms of the output of the microscale models. If the microscale model is molecular dynamics and the macroscale model is continuum hydrodynamics, then this formula is the Irving-Kirkwood formula that expresses stress in terms of the atomistic data from molecular dynamics. If the microscale model is replaced by Brownian dynamics, then this link is replaced by Kramer's expression. From our experience with HMM, we expect that this is a very essential component of any successful multiscale modeling strategy.

How do we formulate the microstructure model on the fibers? Again our experience with HMM suggests that this is usually the most difficult step technically. The basic idea is to constrain the microscale model so that the macroscale variables follow the dynamics on the base manifolds. To overcome the disparity between spatial and temporal scales, we may either rescale the microscale model, or as has been suggested by Weiqing Ren [12], we may formulate the macro and micro simulations using different units. The latter is quite natural since the macro and micro simulations are conducted on different spaces anyway. There is no reason that they need to use the same units. 
In the fiber bundle dynamics models that we have formulated so far, the macroscale data is obtained from the instantaneous results of the microscale model, i.e., we have not made use of the past history of the microscale simulation results. This might be a disadvantage compared with HMM, since the latter tries to make full use of the history of the microscale simulation results by performing sophisticated averaging techniques to extract the needed macroscale data. The same techniques can be used here. In fact, there is no reason why we have to throw away the time history of the microscale results. We can make use of it in the same way as in HMM. For example, we can change (2.7) to

$$
A\left(U_{t}\right)=\int_{t-t_{0}}^{t} K\left(\frac{t-\tau}{t_{0}}\right) \mathcal{A}\left(U_{\tau}, u\left(\cdot ; U_{\tau}\right)\right) \mathrm{d} \tau .
$$

Here $K$ is an averaging kernel as in [9]. This can potentially improve the accuracy of the simulation and reduce statistical error.

The practical aspects of these issues are discussed in [12] and [20].

It should also be remarked that the fiber bundle model is only useful when there is a separation of scales in the system, in which case the local microstructure can be described by the local fiber. In these cases, the fiber bundle model can be used as an effective tool to describe the effective macroscopic model. From an algorithmic viewpoint, it should be considered as an alternative to HMM. Compared with HMM, it has the advantage of being seamless, thereby eliminating some of the most difficult steps in HMM, the steps of going back and forth between macro- and micro-states. It should not be regarded as a closure procedure for systems without scale separation.

Acknowledgement. We are grateful to Weiqing Ren and Eric Vanden-Eijnden for very helpful discussions. This work is partially supported by ONR grant N0001401-1-0674 and NSF grant DMS-0407866.

\section{REFERENCES}

[1] F.F. Abraham, J.Q. Broughton, N. Bernstein and E. Kaxiras, Concurrent coupling of length scales: methodology and application, Phys. Rev. B, 60, 2391-2402, 1999.

[2] R.B. Bird, O. Hassager, R.C. Armstrong and C.F. Curtiss, Dynamics of Polymeric Liquids; Vol. 2: Kinetic Theory, 2nd edition, New York, 1987.

[3] X. Blanc, C. Le Bris and P.L. Lions, From molecular models to continuum mechanics, Arch. Ration. Mech. Anal., 164, 341-381, 2002.

[4] A. Brandt, Multiscale scientific computation: review 2001, in Multiscale and Multiresolution Methods, Lect. Notes Comput. Sci. Eng., Springer, Berlin, 20, 3-95, 2002.

[5] R. Car and M. Parrinello, Unified approach for molecular dynamics and density-functional theory, Phys. Rev. Lett., 55, 2471-2474, 1985.

[6] I. Catto, C. Le Bris and P.L. Lions, The Mathematical Theory of Thermodynamic Limits: Thomas-Fermi Type Models, Clarendon Press, Oxford, 1998.

[7] M. Doi and S.F. Edwards, The Theory of Polymer Dynamics, Oxford University Press, Oxford, 1986.

[8] W. E, B. Engquist, X. Li, W. Ren and E. Vanden-Eijnden, Heterogeneous multiscale methods: a review, Commun. Comput. Phys., 2, 367-450, 2007.

[9] W. E and B. Engquist, The heterogeneous multiscale methods, Commun. Math. Sci., 1, 87-132, 2003.

[10] W. E and B. Engquist, Multiscale modeling and computation, Notices Amer. Math. Soc., 50, 1062-1070, 2003.

[11] W. E and J. Lu, The continuum limit and QM-continuum approximation of quantum mechanical models of solids. Commun. Math. Sci., 5, 679-696, 2007.

[12] W. E, J. Lu, W. Ren and E. Vanden-Eijnden, Seamless multiscale modeling via dynamics on fiber bundles, in preparation. 
[13] W. E and P.B. Ming, Cauchy-Born rule and the stability of crystalline solids: dynamic problems, Acta Math. Appl. Sin. Engl. Ser., in press.

[14] W. E and P.B. Ming, Cauchy-Born rule and the stability of crystalline solids: static problems, Arch. Ration. Mech. Anal., 183, 241-297, 2007.

[15] W. E, The heterogeneous multiscale methods, Proceedings of the 2005 AIChE annual meeting, Cincinnati, 2005.

[16] M.Z. Guo, G.C. Papanicolaou and S.R.S. Varadhan, Nonlinear diffusion limit for a system with nearest neighbor interaction, Commun. Math. Phys., 118, 31-59, 1988.

[17] I.G. Kevrekidis, C.W. Gear, J.M. Hyman, P.G. Kevrekidis, O. Runborg and C. Theodoropoulos, Equation-free, coarse-grained multiscale computation: enabling microscopic simulators to perform system-level analysis, Commun. Math. Sci., 1, 715-762, 2003.

[18] T. Li and P. Zhang, Mathematical analysis of multi-scale models of complex fluids, Commun. Math. Sci., 5, 1-51, 2007.

[19] E.H. Lieb and B. Simon, The Thomas-Fermi theory of atoms, molecules and solids, Adv. Math., 23, 22-116, 1977.

[20] W. Ren, Seamless multiscale modeling of complex fluids using fiber bundle dynamics, preprint.

[21] G.A. Pavliotis and A.M. Stuart, Multiscale Methods: Averaging and Homogenization, in preparation.

[22] E. Vanden-Eijnden, On projective integration methods and HMM-like integrators for systems with multiple time scales, Commun. Math. Sci.,5, 495-505, 2007.

[23] H.T. Yau, Relative entropy and hydrodynamics of Ginzbug-Landau models, Lett. Math. Phys., 22, 63-80, 1991. 